\title{
Valorization of agro-industrial wastes towards the production of rhamnolipids
}

\author{
Eduardo J. Gudiña ${ }^{a, *, 1}$, Ana I. Rodrigues ${ }^{\mathrm{a}, 1}$, Victor de Freitas ${ }^{\mathrm{b}}$, Zélia Azevedo ${ }^{\mathrm{b}}$, José A. Teixeira ${ }^{\mathrm{a}}$, \\ Lígia R. Rodrigues ${ }^{a}$ \\ ${ }^{a}$ CEB - Centre of Biological Engineering, University of Minho, 4710-057 Braga, Portugal \\ ${ }^{\mathrm{b}}$ REQUIMTE/LAQV - Research Unit, Faculty of Science, Porto University, Rua do Campo Alegre, 4169-007 Porto, Portugal
}

\section{H I G H L I G H T S}

- Oil mill wastewater (OMW) was evaluated as an inductor of rhamnolipid production.

- A culture medium containing corn steep liquor, molasses and OMW was designed.

- Pseudomonas aeruginosa \#112 produced $5.1 \mathrm{~g}$ rhamnolipid/1 using that medium.

- The critical micelle concentration of the purified rhamnolipids was $13 \mathrm{mg} / \mathrm{l}$.

- This medium represents a sustainable alternative for rhamnolipid production.

\section{A R T I C L E I N F O}

\section{Article history:}

Received 25 January 2016

Received in revised form 5 April 2016

Accepted 6 April 2016

Available online 8 April 2016

\section{Keywords:}

Oil mill wastewater

Corn steep liquor

Sugarcane molasses

Rhamnolipid

Pseudomonas aeruginosa

\begin{abstract}
A B S T R A C T
In this work, oil mill wastewater (OMW), a residue generated during olive oil extraction, was evaluated as an inducer of rhamnolipid production. Using a medium containing as sole ingredients corn steep liquor $(10 \%, \mathrm{v} / \mathrm{v})$, sugarcane molasses $(10 \%, \mathrm{w} / \mathrm{v})$ and OMW $(25 \%, \mathrm{v} / \mathrm{v})$, Pseudomonas aeruginosa \#112 produced 4.5 and $5.1 \mathrm{~g}$ of rhamnolipid per liter in flasks and reactor, respectively, with critical micelle concentrations as low as $13 \mathrm{mg} / \mathrm{l}$. Furthermore, in the medium supplemented with OMW, a higher proportion of more hydrophobic rhamnolipid congeners was observed comparing with the same medium without OMW. OMW is a hazardous waste which disposal represents a serious environmental problem; therefore, its valorization as a substrate for the production of added-value compounds such as rhamnolipids is of great interest. This is the first report of rhamnolipid production using a mixture of these three agro-industrial by-products, which can be useful for the sustainable production of rhamnolipids.
\end{abstract}

() 2016 Elsevier Ltd. All rights reserved.

\section{Introduction}

Surfactants are an important class of amphiphilic compounds. Their hydrophobic and hydrophilic moieties tend to distribute at the interfaces between fluid phases with different polarities, reducing the surface and interfacial tensions. As a result, these compounds facilitate the formation of emulsions, dispersions and foam, making them essential in applications that require emulsification, lubrication, foaming, solubilization of immiscible compounds or phase dispersion. Their uses include the formulation of

\footnotetext{
* Corresponding author at: CEB - Centre of Biological Engineering, University of Minho, Campus de Gualtar, 4710-057 Braga, Portugal.

E-mail address: egudina@deb.uminho.pt (E.J. Gudiña).

1 These authors contributed equally to this work.
}

cleaning products and detergents, applications in food processing, agriculture, environmental remediation or tertiary oil recovery, among others (Mnif and Ghribi, 2015).

The global market of surfactants is continuously growing. Their total worldwide production is estimated to be over 15 million tons per year, and it is expected to reach about 24 million tons and 42,120 million USD per year by 2020 (MarketsandMarkets, 2015). Most of the surfactants available nowadays are derived from petrochemical sources, which besides contributing to environmental pollution due to their low biodegradability, consume nonrenewable resources. Surface-active compounds synthesized by microorganisms (biosurfactants) display a similar or better performance when compared with chemical surfactants, besides exhibiting higher biodegradability and lower toxicity, being an environmentally friendly alternative to conventional synthetic surfactants (Pereira et al., 2013; Vaz et al., 2012). 
Among biosurfactants, rhamnolipids have attracted a pronounced attention in the last years. Rhamnolipids are a class of glycolipid biosurfactants produced mainly by Pseudomonas aeruginosa strains, which consist of one or two rhamnose molecules linked to one or two $\beta$-hydroxy fatty acids (Henkel et al., 2012; Kiran et al., 2015; Lovaglio et al., 2015). Besides their ability to reduce the surface/interfacial tension and their emulsifying activity, rhamnolipids exhibit additional properties when compared with chemical surfactants, including antimicrobial and antitumor activities (Bharali et al., 2013; Christova et al., 2013), which expands the spectrum of their potential applications, increasing their value. Examples of companies that commercialize rhamnolipids are AGAE Technologies $^{\circledR}$, USA (www.agaetech.com); Rhamnolipid Companies Inc., USA (www.rhamnolipid.com); Jeneil Biotech Inc., USA (www.jeneilbiotech.com); and Urumqi Unite Bio-Technology Co. Ltd., China (www.unite-xj.en.alibaba.com).

However, one of the main limitations associated to the commercialization of biosurfactants is their high production costs when compared with synthetic surfactants. That can be partially overcome by using inexpensive substrates, such as agroindustrial by-products or wastes (Banat et al., 2014; Gudiña et al., 2015; Henkel et al., 2012). Furthermore, if the residues used are environmentally hazardous and their disposal is problematic, their valorization as substrates for biosurfactants production represents an additional advantage.

Olive oil is one of the most widely consumed edible oils, being the leading producers Spain, Italy, Greece and Portugal, which account for approximately $98 \%$ of its worldwide production (Dermeche et al., 2013; Gonçalves et al., 2009). During its extraction, two different wastes are generated, namely a solid residue and an effluent known as olive mill wastewater (OMW). The main components of OMW, apart from water (83\% to 94\%), are lipids, carbohydrates, phenolic compounds, organic acids, tannins, pectins and minerals (Dermeche et al., 2013; Meksi et al., 2012). The composition of OMW varies, both qualitatively and quantitatively, according to the olive variety, the climate conditions, the agronomic practices and especially the technology used to extract the oil from the olives, which can be a discontinuous pressing process or a continuous (two- or three-phase) extraction process. In Portugal, the most common is the continuous three-phase extraction process, which uses more water to extract the oil when compared with the two-phase process, and consequently generates higher amounts of OMW (Gonçalves et al., 2009). It is estimated that about 30 million $\mathrm{m}^{3}$ of OMW are produced each year in the Mediterranean countries (Dermeche et al., 2013; Meksi et al., 2012). The generation of this residue is seasonal, associated to the production of olive oil, which in Portugal usually takes place between December and February. However, in other Mediterranean countries its production can comprise a longer period (from October to March) (Dermeche et al., 2013; Gonçalves et al., 2012). OMW is a significant pollutant and its disposal constitutes an environmental problem, due to its low degradability and its toxicity against microorganisms, plants and aquatic ecosystems (Dermeche et al., 2013).

In this work, OMW was evaluated as an inducer of rhamnolipid production by a $P$. aeruginosa strain, both in flasks and reactor. Its inductive effect was evaluated by supplementing a culture medium containing corn steep liquor and sugarcane molasses with OMW at different concentrations. Furthermore, the main rhamnolipid congeners produced were characterized.

\section{Material and methods}

\subsection{Bacterial strain}

The bacterial strain $P$. aeruginosa \#112 was used for rhamnolipid production. This strain, isolated from a crude oil sample obtained from a Brazilian oil field, was previously reported as a promising rhamnolipid producer (Gudiña et al., 2015). It was stored at $-80^{\circ} \mathrm{C}$ in Luria Bertani (LB) medium supplemented with $20 \%(\mathrm{v} / \mathrm{v})$ of glycerol. The composition of LB medium was ( $\mathrm{g} / \mathrm{l})$ : $\mathrm{NaCl} 10.0$; tryptone 10.0; yeast extract 5.0; $\mathrm{pH} 7.0$.

\subsection{Agro-industrial residues}

Sugarcane molasses and corn steep liquor (CSL) were kindly provided by RAR: Refinarias de Açúcar Reunidas, S.A. (Portugal) and COPAM: Companhia Portuguesa de Amidos, S.A. (Portugal), respectively. OMW was obtained from an olive oil mill located in the north of Portugal, which uses a continuous three-phase centrifugation process for olive oil extraction. All the residues were stored at $4{ }^{\circ} \mathrm{C}$ until use. The concentration of total carbohydrates and protein in these residues were determined using the phenolsulfuric and Lowry methods, respectively (Dubois et al., 1956; Lowry et al., 1951). Furthermore, OMW was characterized in terms of $\mathrm{pH}$, total solids, total phenols, total lipids and long-chain fatty acids composition, using methodologies described elsewhere (Amaral et al., 2012; Gonçalves et al., 2009, 2012). Total solids were determined by oven drying $\left(105^{\circ} \mathrm{C}\right)$ to constant weight. Total phenols were evaluated by the Folin-Ciocalteu method and expressed as $\mathrm{mg}_{\text {caffeic }}$ acid/l. Lipids were extracted with diethyl ether, in a Soxtec $^{\mathrm{TM}} 8000$ extraction unit (FOSS, Hillerød, Denmark). Longchain fatty acids were determined by gas chromatography.

\subsection{Rhamnolipid production}

OMW was evaluated as an inducer of rhamnolipid production. For that purpose, different culture media were prepared containing CSL $(10 \%, v / v)$ and sugarcane molasses $(10 \%, \mathrm{w} / \mathrm{v})$, supplemented with OMW at concentrations between $5 \%$ and $25 \%(\mathrm{v} / \mathrm{v})$. All the media were adjusted to $\mathrm{pH}$ 7.0.

Rhamnolipid production by $P$. aeruginosa \#112 was studied in $500 \mathrm{ml}$ flasks containing $200 \mathrm{ml}$ of the different culture media. Each flask was inoculated with $1 \%(\mathrm{v} / \mathrm{v})$ of a pre-culture of $P$. aeruginosa \#112 grown overnight in LB medium, and after that they were incubated at $37^{\circ} \mathrm{C}$ and $180 \mathrm{rpm}$. Samples were taken at different time points during the fermentation in order to evaluate the production of rhamnolipids. The samples were centrifuged $\left(10,000 \times \mathrm{g}, 20 \mathrm{~min}, 20^{\circ} \mathrm{C}\right)$ and the cell-free supernatants were used to measure the surface tension (ST) and to determine the emulsifying activity $\left(E_{24}\right)$, as described below. Whenever required, the cell-free supernatants were diluted with demineralized water and the corresponding surface tensions were measured, in order to study the evolution of biosurfactant production. The cultures were maintained until the maximum rhamnolipid production was achieved (according to the surface tension values and the emulsifying indexes). All the experiments were performed in triplicate.

\subsection{Rhamnolipid recovery}

The rhamnolipids produced by $P$. aeruginosa \#112 were recovered from the cell-free supernatants obtained at the end of the different fermentations by adsorption chromatography using the polystyrene resin Amberlite XAD-2 (Sigma-Aldrich Co., USA) as previously described by Gudiña et al. (2015). Rhamnolipid production was determined as dry weight of the freeze-dried purified products.

\subsection{Surface-activity determination}

The surface tension measurements were performed according to the Ring method as described elsewhere (Gudiña et al., 2012) 
using a KRÜSS K20 Tensiometer (KRÜSS GmbH, Germany) equipped with a $1.9 \mathrm{~cm}$ De Noüy platinum ring at room temperature $\left(25^{\circ} \mathrm{C}\right)$. All the measurements were performed in triplicate.

\subsection{Emulsifying activity determination}

The emulsifying activity was determined using $n$-hexadecane, as described elsewhere (Gudiña et al., 2012). All the emulsification indexes were determined in triplicate.

\subsection{Determination of the critical micelle concentration $(\mathrm{cmc})$}

The critical micelle concentrations (cmcs) were calculated as described by Gudiña et al. (2012), using the freeze-dried rhamnolipids produced by $P$. aeruginosa \#112 in the different culture media, dissolved in demineralized water at different concentrations. All the measurements were performed in triplicate.

\subsection{Rhamnolipid production by P. aeruginosa \#112 in reactor}

A 51 reactor (BIOSTAT ${ }^{\circledR}$ A Fermentor, B. Braun Biotech International $\mathrm{GmbH}$, Germany), equipped with agitation, temperature, $\mathrm{pO}_{2}$ and $\mathrm{pH}$ online measurement and control was used. The experiments were performed with 21 working volume at $37^{\circ} \mathrm{C}$. The reactor was inoculated with $1 \%(\mathrm{v} / \mathrm{v})$ of a pre-culture of $P$. aeruginosa \#112 grown overnight in LB medium at $37^{\circ} \mathrm{C}$ and $180 \mathrm{rpm}$. The effect of different agitation (200-450 rpm) and airflow (0.2$1 \mathrm{vvm}$ ) rates on rhamnolipid production was studied. Air was injected in the top of the reactor, without contacting the culture medium, to avoid the formation of foam. The fermentations were conducted as batch cultivations without $\mathrm{pH}$ control. In order to evaluate bacterial growth and rhamnolipid production, samples were taken at different time points during the fermentation. The fermentations were maintained until the maximum rhamnolipid production was achieved (according to the surface tension values and the emulsifying indexes). Surface tension and emulsifying activity measurements, rhamnolipid recovery and $\mathrm{cmc}$ calculation were performed as described above. Bacterial growth was determined by measuring the optical density at $600 \mathrm{~nm}$, and biomass concentration ( $g$ dry weight/l) was calculated using a calibration curve.

\subsection{Identification of rhamnolipid congeners}

For chemical composition analysis, the freeze-dried biosurfactant samples were prepared in two different ways. First they were dissolved in methanol, centrifuged $(10,000 \times g, 5 \mathrm{~min})$, and the final solution was analyzed by direct flow injection. Secondly, the samples were dissolved in water at a concentration of $10 \mathrm{mg} / \mathrm{ml}$ and extracted with a chloroform/methanol mixture $(2: 1, \mathrm{v} / \mathrm{v})$ to achieve a final chloroform/methanol/water ratio of $8: 4: 3(\mathrm{v} / \mathrm{v} / \mathrm{v})$. The final mixture was centrifuged $(10,000 \times g, 5 \mathrm{~min})$, and the resulting organic layer analyzed in the same way.

The samples were analyzed by electrospray ionization (ESI) mass spectrometry (MS). An aliquot of each sample was injected by flow injection analysis (FIA) into an LTQ-Orbitrap XL mass spectrometer (ThermoFisher Scientific, Waltham, MA, USA). The sample was pumped at $5 \mu \mathrm{l} / \mathrm{min}$ using nitrogen as sheath gas at a flow of 5 (arbitrary unit system). The electrospray conditions were as follows: $3 \mathrm{kV}$ for the spray voltage; $270^{\circ} \mathrm{C}$ for the capillary temperature; the tube lens was established at $100 \mathrm{~V}$ under optimum analysis conditions. All the organic solvents used were of analytical grade or mass spectrometry grade for Orbitrap analysis, and were purchased from Panreac (Castellar del Vallés, Barcelona, Spain). Milli-Q water was obtained from a water purification system (Millipore, Millerica, MA, USA). Trifluoroacetic acid (HPLC grade) was purchased from Sigma-Aldrich (USA).

\section{Results and discussion}

\subsection{Effect of OMW on rhamnolipid production by P. aeruginosa \#112}

Previously, we developed the culture medium CSLM for rhamnolipid production, containing as sole ingredients the agroindustrial by-products CSL $(10 \%, v / v)$ and sugarcane molasses $(10 \%, \mathrm{w} / \mathrm{v})$. Using that medium, the isolate $P$. aeruginosa \#112 produced $3.2 \mathrm{~g}$ of rhamnolipids per liter, with a $\mathrm{cmc}$ of $50 \mathrm{mg} / \mathrm{l}$ (Gudiña et al., 2015). In this work, the agro-industrial waste OMW (which composition is summarized in Table 1) was evaluated as an inducer of rhamnolipid production. Although the composition of OMW depends on several factors as previously explained, the results herein showed are in accordance with previous characterizations of OMW obtained from the same region (Amaral et al., 2012; Gonçalves et al., 2009, 2012).

As it can be seen in Table 1, OMW is a source of long-chain fatty acids, including palmitic $\left(\mathrm{C}_{16}\right)$, stearic $\left(\mathrm{C}_{18}\right)$, oleic $\left(\mathrm{C}_{18: 1}\right)$ and linoleic acid $\left(C_{18: 2}\right)$, being oleic acid the most abundant $(3.3 \mathrm{~g} / \mathrm{l})$. It has been reported that using a combination of carbohydrates and long-chain fatty acids, the rhamnolipid production by $P$. aeruginosa strains is higher than using carbohydrates as a single carbon source (Zhang et al., 2012, 2014). Therefore, OMW can be used as an inexpensive source of long-chain fatty acids to stimulate the production of rhamnolipids. Given the good results obtained previously using the medium CSLM, rhamnolipid production by $P$. aeruginosa \#112 was assayed using the same medium but now supplemented with OMW at different concentrations (5-25\%,v/v). The results obtained are shown in Table 2.

The surface tension of the different media was around $50 \mathrm{mN} /$ $\mathrm{m}$. As can be seen from Table 2, a considerable surface tension reduction was observed at the end of the different fermentations. With the medium CSLM without OMW, the maximum rhamnolipid production was achieved after $144 \mathrm{~h}$ of growth; however, in all the media supplemented with OMW, the maximum rhamnolipid production was achieved at $168 \mathrm{~h}$. Although the surface tension values obtained at the end of the fermentation with the different media were very similar (around $31 \mathrm{mN} / \mathrm{m}$ ), as the OMW concentration increased (from 5\% to 15\%) the amount of rhamnolipid produced also increased, reaching $5.030 \pm 0.221 \mathrm{~g} / \mathrm{l}$ for an OMW concentration of $15 \%$. At the same time, the $\mathrm{cmc}$ of the rhamnolipids produced decreased, from $50 \mathrm{mg} / \mathrm{l}$ up to $34 \mathrm{mg} / \mathrm{l}$. The media containing $20 \%$ and $25 \%$ of OMW led to the production of lower amounts of rhamnolipids when compared with the media containing $10 \%$ and $15 \%$ of OMW, although with lower $\mathrm{cmc}$ values (14$15 \mathrm{mg} / \mathrm{l}$ ) (Table 2). Rhamnolipids are usually produced as mixtures of different compounds (mono- and di-rhamnolipids, containing one or two fatty acids with different chain lengths) (AbdelMawgoud et al., 2010). The differences observed in the $\mathrm{cmc}$ values herein obtained can be due to the different purity of the products recovered, or to differences in the qualitative and/or quantitative composition of the rhamnolipid mixtures produced, as it will be later discussed. In all the cases, the purified rhamnolipids reduced the surface tension of demineralized water from $72 \mathrm{mN} / \mathrm{m}$ up to $27 \mathrm{mN} / \mathrm{m}$ at concentrations equal or higher than their $\mathrm{cmc}$. Regarding the emulsifying activity, the best results were obtained for the culture media supplemented with OMW at concentrations between $10 \%$ and $25 \%$ (Table 2 ).

Several authors reported the use of different agro-industrial wastes or by-products as substrates for the production of rhamnolipids by different Pseudomonas sp. strains, including orange peels, oil wastes or molasses. Using those substrates, the surface tension of the culture medium was reduced up to $30-33 \mathrm{mN} / \mathrm{m}$, and emulsifying indexes between $59 \%$ and $84 \%$ were obtained; the amounts of rhamnolipid produced were between 5 and $14 \mathrm{~g} / \mathrm{l}$, with $\mathrm{cmcs}$ between 27 and $120 \mathrm{mg} / \mathrm{l}$ (Aparna et al., 2012; Benincasa and 
Table 1

Characterization of OMW used in this work. Results represent the average \pm standard deviation of three independent measurements.

\begin{tabular}{lr}
\hline Parameter & \multicolumn{1}{c}{ Value } \\
\hline $\mathrm{pH}$ & $5.1 \pm 0.1$ \\
Total solids $(\mathrm{g} / \mathrm{l})$ & $59.8 \pm 3.0$ \\
Total phenols $(\mathrm{g} / \mathrm{l})$ & $2.6 \pm 0.1$ \\
Total carbohydrates $(\mathrm{g} / \mathrm{l})$ & $11.7 \pm 0.9$ \\
Total proteins $(\mathrm{g} / \mathrm{l})$ & $0.45 \pm 0.05$ \\
Lipids $(\mathrm{g} / \mathrm{l})$ & $4.8 \pm 0.3$ \\
Long-chain fatty acids $(\mathrm{mg} / \mathrm{l})$ & \\
Palmitic acid $\left(\mathrm{C}_{16}\right)$ & $513 \pm 32$ \\
Stearic acid $\left(\mathrm{C}_{18}\right)$ & $129 \pm 12$ \\
Oleic acid $\left(\mathrm{C}_{18: 1}\right)$ & $3387 \pm 89$ \\
Linoleic acid $\left(\mathrm{C}_{18: 2}\right)$ & $284 \pm 21$ \\
\hline
\end{tabular}

Accorsini, 2008; George and Jayachandran, 2009; Haba et al., 2003; Lan et al., 2015; Nitschke et al., 2005). However, in most of these cases, the culture medium was supplemented with additional carbon or nitrogen sources, salts or mineral elements, which increases the production costs. Furthermore, in these reports the rhamnolipid yields were calculated indirectly using colorimetric methods such as the orcinol assay, which overestimates between 5 and 9 times the real concentration of rhamnolipids in the samples, as discussed by Perfumo et al. (2013).

Ramírez et al. (2015) reported the production of rhamnolipids by a $P$. aeruginosa strain using 'alperujo' (a solid residue generated during olive oil production using the two-phase extraction process) as the sole carbon source $(10 \%, \mathrm{w} / \mathrm{v})$. However, the culture medium was supplemented with additional nutrients, and the rhamnolipid yield obtained was $0.191 \mathrm{~g} / \mathrm{l}$, which is considerably lower when compared with the results herein reported.

Supplementing the low-cost culture medium CSLM (which value is about $0.024 € / 1$ ) with OMW at different concentrations resulted not only in the production of higher amounts of rhamnolipids, but also in the production of more efficient rhamnolipid mixtures (i.e. with lower $\mathrm{cmc}$ values). To the best of our knowledge this is the first time that rhamnolipid production using a combination of CSL, sugarcane molasses and OMW is reported. Furthermore, these substrates were used without any previous treatment and without additional supplements. Taking into account the amount of rhamnolipids produced and their $c m c s$, it can be concluded that the medium CSLM supplemented with $25 \%$ of OMW offered the best results.

In $P$. aeruginosa, the expression of the genes $r h l A$ and $r h l B$, involved in the biosynthesis of rhamnolipids, is increased up to 600 times when long-chain fatty acids (including oleic, linoleic and stearic acid) are used as co-substrates together with glucose, when compared with the use of glucose as the sole carbon source (Zhang et al., 2012). Furthermore, when carbohydrates are the sole carbon source, the precursors for the synthesis of the lipid moiety of rhamnolipids are provided only by de novo fatty acid synthesis. However, in the presence of carbohydrates and long-chain fatty acids, they are supplied by de novo fatty acid synthesis and $\beta$ oxidation, which results in a higher production of lipid precursors for rhamnolipid biosynthesis (Zhang et al., 2014). As a result of both inductive processes, rhamnolipid production is increased up to two times when the culture medium contains carbohydrates and long-chain fatty acids (Zhang et al., 2012, 2014). This could explain the inductive effect of OMW on the rhamnolipid production when added to the culture medium CSLM.

\subsection{Rhamnolipid production by P. aeruginosa \#112 in reactor}

The media CSLM and CSLM supplemented with 25\% of OMW were evaluated for rhamnolipid production in a 51 reactor. Different agitation (200-450 rpm) and aeration $(0.2-1 \mathrm{vvm})$ rates were assayed, and the best results were obtained at $350 \mathrm{rpm}$ and $0.5 \mathrm{vvm}$, with the air injected in the top of the reactor. With this foam-free production approach, only a thin layer of foam was formed in the upper phase of the medium, similar to that formed in the fermentations performed in flasks. The results obtained are summarized in Table 3 and Fig. 1. With both media, the surface tension reductions obtained were higher when compared with the assays performed in flaks; however, the emulsifying indexes were slightly lower. Using the medium CSLM without OMW, the amount of rhamnolipid produced in the reactor was lower when compared with the assays performed in flasks, although it exhibited a lower $\mathrm{cmc}$ (i.e. it was more efficient). Regarding the medium CSLM supplemented with 25\% of OMW, the amount of rhamnolipid produced in the reactor was higher when compared with the assays performed in flasks, and their cmcs were similar.

Fig. 1 shows the evolution of cell growth, surface tension and emulsifying activity in these assays. In the case of the medium containing OMW (Fig. 1B), growth and rhamnolipid production started later when compared with the medium CSLM (Fig. 1A), which can be probably due to the toxic effect of some components of OMW (e.g. phenolic compounds). With both media, the surface tension of the cell-free supernatants decreased quickly after the lag phase to values around $30 \mathrm{mN} / \mathrm{m}$ and then remained almost constant until the end of the fermentation. This means that the amount of rhamnolipids present in the medium was equal or higher than the $c m c$. Consequently, it was necessary to dilute the cell-free supernatants to study the evolution of rhamnolipid production along the fermentations. The surface tension of the 100 times diluted cell-free supernatant continued decreasing until the end of the fermentation in both media, thus indicating a continuous production of rhamnolipids (Fig. 1). However, the emulsifying indexes measured in the supernatants without dilution continued increasing in both media until the end of the fermentation, meaning that a higher concentration of rhamnolipids is necessary to achieve a high emulsifying activity when compared with that necessary to reduce the surface tension. Regarding the medium CSLM, biomass concentration reached its maximum after $90 \mathrm{~h}$ $(3.686 \pm 0.105 \mathrm{~g}$ dry weight/l), while the highest rhamnolipid

Table 2

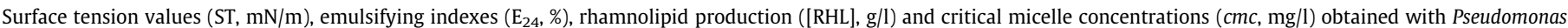

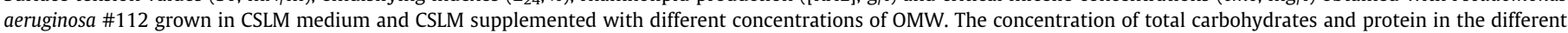
culture media is also given. Results represent the average of three independent experiments \pm standard deviation.

\begin{tabular}{|c|c|c|c|c|c|c|c|}
\hline Medium & [Carbohydrates] (g/l) & [Protein] $(\mathrm{g} / \mathrm{l})$ & Time (h) & $\mathrm{ST}(\mathrm{mN} / \mathrm{m})$ & $\mathrm{E}_{24}(\%)$ & [RHL] (g/l) & $\mathrm{cmc}(\mathrm{mg} / \mathrm{l})$ \\
\hline CSLM & 56.5 & 0.56 & 144 & $31.4 \pm 0.1$ & $59.0 \pm 1.5$ & $3.194 \pm 0.245$ & 50 \\
\hline CSLM + 5\% OMW & 57.1 & 0.58 & 168 & $31.3 \pm 0.3$ & $58.2 \pm 2.5$ & $3.758 \pm 0.176$ & 38 \\
\hline CSLM $+10 \%$ OMW & 57.7 & 0.60 & 168 & $31.4 \pm 0.2$ & $63.5 \pm 1.4$ & $4.660 \pm 0.102$ & 36 \\
\hline CSLM + 15\% OMW & 58.2 & 0.63 & 168 & $31.3 \pm 0.2$ & $64.0 \pm 2.1$ & $5.030 \pm 0.221$ & 34 \\
\hline CSLM $+20 \%$ OMW & 58.8 & 0.65 & 168 & $31.0 \pm 0.1$ & $63.9 \pm 2.0$ & $3.848 \pm 0.190$ & 15 \\
\hline CSLM $+25 \%$ OMW & 59.4 & 0.67 & 168 & $31.0 \pm 0.1$ & $64.0 \pm 0.5$ & $4.526 \pm 0.105$ & 14 \\
\hline
\end{tabular}

CSLM: corn steep liquor $(10 \%, \mathrm{v} / \mathrm{v})+$ sugarcane molasses $(10 \%, \mathrm{w} / \mathrm{v})$. 
Table 3

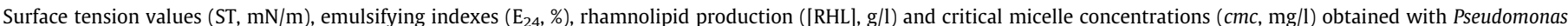

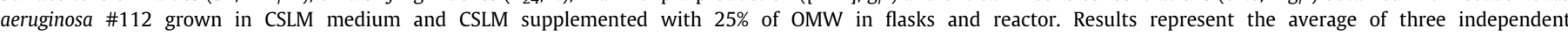
experiments \pm standard deviation.

\begin{tabular}{|c|c|c|c|c|c|c|}
\hline Medium & & Time (h) & $\mathrm{ST}(\mathrm{mN} / \mathrm{m})$ & $\mathrm{E}_{24}(\%)$ & {$[\mathrm{RHL}](\mathrm{g} / \mathrm{l})$} & $\mathrm{cmc}(\mathrm{mg} / \mathrm{l})$ \\
\hline \multirow[t]{2}{*}{ CSLM } & Flask & 144 & $31.4 \pm 0.1$ & $59.0 \pm 1.5$ & $3.194 \pm 0.245$ & 50 \\
\hline & Reactor & 96 & $29.0 \pm 0.2$ & $54.2 \pm 2.0$ & $2.236 \pm 0.103$ & 30 \\
\hline \multirow[t]{2}{*}{ CSLM + 25\% OMW } & Flask & 168 & $31.0 \pm 0.1$ & $64.0 \pm 0.5$ & $4.526 \pm 0.105$ & 14 \\
\hline & Reactor & 168 & $29.2 \pm 0.2$ & $58.4 \pm 1.0$ & $5.124 \pm 0.125$ & 13 \\
\hline
\end{tabular}

CSLM: corn steep liquor $(10 \%, \mathrm{v} / \mathrm{v})+$ sugarcane molasses $(10 \%, \mathrm{w} / \mathrm{v})$.

production (according to the surface tension of 100 times diluted supernatant) was achieved at $96 \mathrm{~h}$. In the case of the medium CSLM supplemented with $25 \%$ of OMW, the highest biomass concentration was achieved at $162 \mathrm{~h}(3.934 \pm 0.110 \mathrm{~g}$ dry weight/l) and the highest rhamnolipid production at $168 \mathrm{~h}$. In both cases, a parallel relationship was observed between cell growth and rhamnolipid production, which suggests a growth-associated production profile. The same profile was reported for different $P$. aeruginosa strains by other authors (Aparna et al., 2012; Bharali and Konwar, 2011). However, in other cases it has been referred that rhamnolipid production occurs mainly during the stationary growth phase (Haba et al., 2003; Nitschke et al., 2005; Ramírez et al., 2015).

Fermentations performed with biosurfactant-producing microorganisms are usually associated to a severe foam production, which can result in the overflow of the culture medium and
A

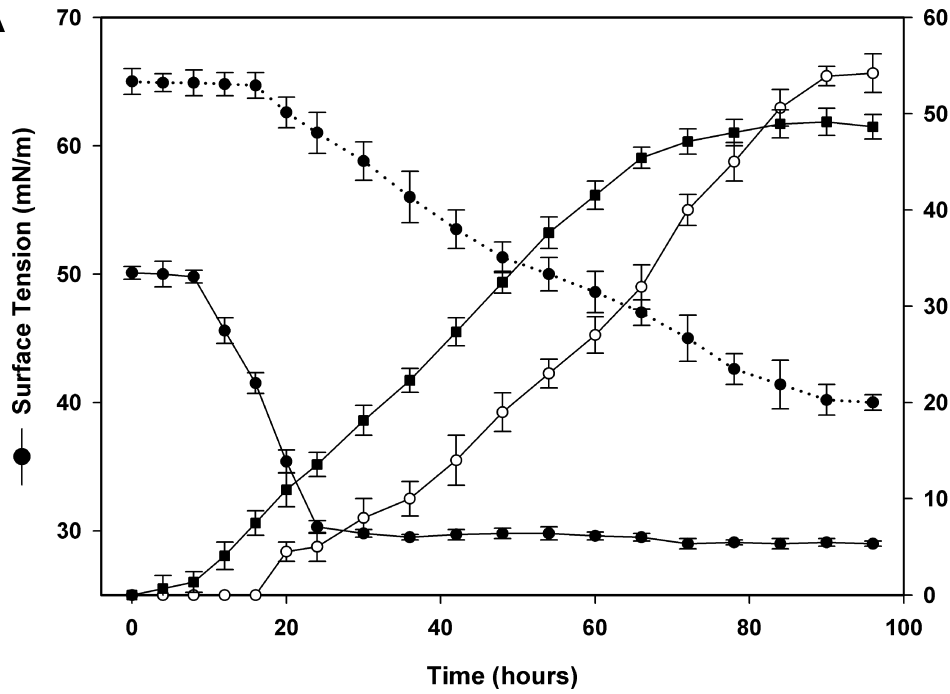

B

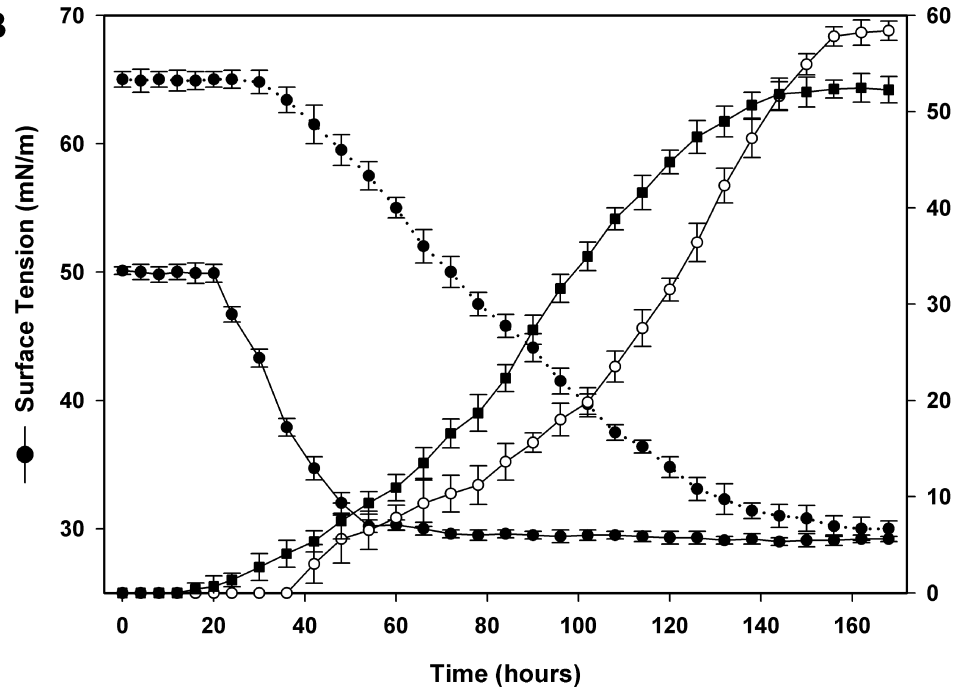

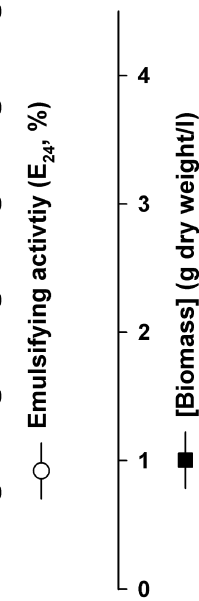

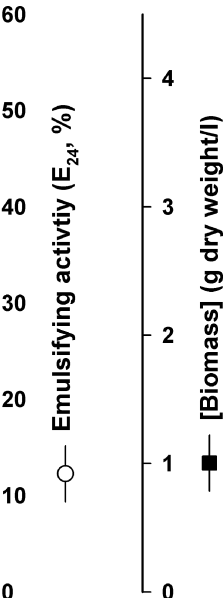

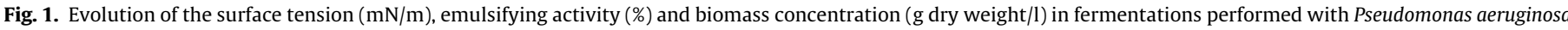

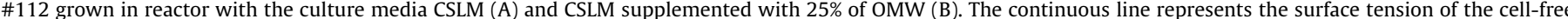

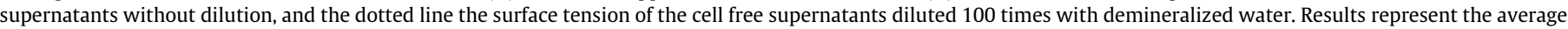
of three independent experiments \pm standard deviation. 
microbial cells. The use of antifoam agents is not attractive from an economic point of view; furthermore, these compounds are difficult to remove in the downstream processing, and can have a negative effect on the biosurfactant production. As an alternative, the foam produced (which contains a high biosurfactant concentration) can be channeled through one of the outputs of the reactor and collected to further extract the biosurfactant, in a process known as foam fractionation. Although this technology has been widely used for biosurfactant production in the laboratory (Sarachat et al., 2010; Willenbacher et al., 2015), it requires a complex design and construction that can affect the scale-up process and its industrial application. Consequently, the application of a foam-free fermentation strategy as described here can be a better alternative.

\subsection{Characterization of rhamnolipid mixtures}

The rhamnolipid mixtures produced by $P$. aeruginosa \#112 in assays performed in flasks and reactor with different media were characterized by mass spectrometry by direct injection in an Orbitrap ESI MS spectrometer operating in the negative mode. The measured accurate mass of the $[\mathrm{M}-\mathrm{H}]^{-}$pseudomolecular ions was matched with exact mass of the molecular structures expected for mono- and di-rhamnolipids, based on other similar studies (Abdel-Mawgoud et al., 2010; Gudiña et al., 2015), and the mass error was calculated (Table 4).

According to Table 5, in all the cases seven different rhamnolipid congeners were identified, including mono- and dirhamnolipids. The most abundant congener was the monorhamnolipid Rha- $\mathrm{C}_{10}-\mathrm{C}_{10}$, followed by the di-rhamnolipid RhaRha- $C_{10}-C_{10}$ and then the mono-rhamnolipid Rha- $C_{10}$, regardless of the culture media used, both in fermentations performed in flasks or in reactor. These results are in agreement with the results obtained in the positive mode in which the sodium adducts of the rhamnolipids Rha- $C_{10}-C_{10}$ and Rha-Rha- $C_{10}-C_{10}$ were also the most abundant ions. The predominant hydroxyl fatty acid was decanoic acid, with trace amounts of octanoic and dodecanoic acids.

The main differences observed between the different culture media were in the relative abundance of some rhamnolipid congeners. In assays performed in flasks, supplementing the medium CSLM with OMW resulted in a reduction in the relative abundance of the mono-rhamnolipid Rha- $\mathrm{C}_{10}$; and with the medium supplemented with $15 \%$ of OMW, a higher relative abundance of the dirhamnolipid Rha-Rha- $\mathrm{C}_{10}-\mathrm{C}_{10}$ was observed when compared with the other two media. Regarding the ratio mono-/di-rhamnolipid, the same value was observed for the media CSLM and CSLM supplemented with $25 \%$ of OMW, whereas the medium CSLM supplemented with $15 \%$ of OMW exhibited a lower proportion of monorhamnolipids.

In assays performed in reactor, using the medium CSLM supplemented with $25 \%$ of OMW, a lower relative abundance of all the dirhamnolipid congeners, as well as the mono-rhamnolipid Rha- $\mathrm{C}_{10}$ was observed when compared with the medium CSLM, which resulted in a higher proportion of mono-rhamnolipids.

It has been previously reported that the constituents of the culture medium exert a significant effect in the composition (both qualitative and quantitative) of the rhamnolipid mixtures produced by $P$. aeruginosa strains (Gudiña et al., 2015; Ismail et al., 2015; Zhang et al., 2014). P. aeruginosa strains produce rhamnolipids as combinations of different congeners (up to 60 different types have been reported), which can be mono- and dirhamnolipids with one or two acyl chains containing $8,10,12$ or 14 carbons, mostly saturated, and, less often, containing one or two double bonds, being the most common Rha- $C_{10}-C_{10}$ and Rha-Rha- $\mathrm{C}_{10}-\mathrm{C}_{10}$ (Abdel-Mawgoud et al., 2010; Aparna et al., 2012; Benincasa and Accorsini, 2008; Bharali and Konwar, 2011; Haba et al., 2003; Ismail et al., 2015; Ramírez et al., 2015; Zhang

Table 4

Rhamnolipid congeners identified as $[\mathrm{M}-\mathrm{H}]^{-}$ions by mass spectrometry in the different rhamnolipid mixtures produced by Pseudomonas aeruginosa \#112.

\begin{tabular}{|c|c|c|c|c|}
\hline Compound & {$[\mathrm{M}-\mathrm{H}]^{-}$measured mass } & {$[\mathrm{M}-\mathrm{H}]^{-}$exact mass } & Elemental composition & Mass error (ppm) \\
\hline Rha- $C_{10}$ & 333.19169 & 333.19188 & $\mathrm{C}_{16} \mathrm{H}_{29} \mathrm{O}_{7}$ & 0.57 \\
\hline Rha-Rha- $C_{10}$ & 479.24981 & 479.24979 & $\mathrm{C}_{22} \mathrm{H}_{39} \mathrm{O}_{11}$ & -0.042 \\
\hline Rha- $C_{10}-C_{10}$ & 503.32234 & 503.32256 & $\mathrm{C}_{26} \mathrm{H}_{47} \mathrm{O}_{9}$ & 0.44 \\
\hline Rha $-C_{10}-C_{12}{ }^{a}$ & 531.35332 & 531.35386 & $\mathrm{C}_{28} \mathrm{H}_{51} \mathrm{O}_{9}$ & 1.02 \\
\hline Rha-Rha- $C_{10}-C_{8}{ }^{b}$ & 621.34942 & 621.34917 & $\mathrm{C}_{30} \mathrm{H}_{53} \mathrm{O}_{13}$ & -0.4 \\
\hline Rha-Rha- $C_{10}-C_{10}$ & 649.38051 & 649.38047 & $\mathrm{C}_{32} \mathrm{H}_{57} \mathrm{O}_{13}$ & -0.06 \\
\hline Rha-Rha- $\mathrm{C}_{10}-\mathrm{C}_{12}{ }^{\mathrm{c}}$ & 677.41140 & 677.41177 & $\mathrm{C}_{34} \mathrm{H}_{61} \mathrm{O}_{13}$ & 0.55 \\
\hline
\end{tabular}

${ }^{\text {a }}$ Or Rha- $\mathrm{C}_{12}-\mathrm{C}_{10}$.

b Or Rha-Rha- $C_{8}-C_{10}$.

c Or Rha-Rha- $\mathrm{C}_{12}-\mathrm{C}_{10}$.

Table 5

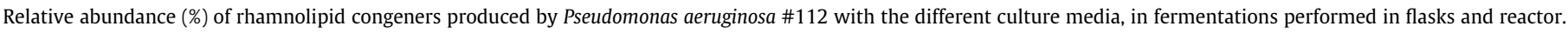

\begin{tabular}{|c|c|c|c|c|c|c|}
\hline \multirow[t]{2}{*}{ Rhamnolipid congener } & \multirow[t]{2}{*}{ MW } & \multicolumn{3}{|l|}{ Flask } & \multicolumn{2}{|c|}{ Reactor } \\
\hline & & CSLM & $\mathrm{CSLM}+15 \% \mathrm{OMW}$ & CSLM + 25\% OMW & CSLM & CSLM + 25\% OMW \\
\hline Rha- $\mathrm{C}_{10}$ & 334.19915 & $38 \%$ & $32 \%$ & $29 \%$ & $38 \%$ & $30 \%$ \\
\hline Rha- $C_{10}-C_{10}$ & 504.32983 & $100 \%$ & $100 \%$ & $100 \%$ & $100 \%$ & $100 \%$ \\
\hline Rha- $\mathrm{C}_{10}-\mathrm{C}_{12}{ }^{\mathrm{a}}$ & 532.36113 & $12 \%$ & $12 \%$ & $13 \%$ & $12 \%$ & $12 \%$ \\
\hline Rha-Rha- $\mathrm{C}_{10}$ & 480.25706 & $12 \%$ & $14 \%$ & $11 \%$ & $20 \%$ & $10 \%$ \\
\hline Rha-Rha- $\mathrm{C}_{10}-\mathrm{C}_{8}{ }^{\mathrm{b}}$ & 622.35644 & $4 \%$ & $4 \%$ & $4 \%$ & $14 \%$ & $2 \%$ \\
\hline Rha-Rha- $C_{10}-C_{10}$ & 650.38774 & $60 \%$ & $66 \%$ & $58 \%$ & $78 \%$ & $32 \%$ \\
\hline Rha-Rha- $\mathrm{C}_{10}-\mathrm{C}_{12}{ }^{\mathrm{C}}$ & 678.41904 & $14 \%$ & $14 \%$ & $12 \%$ & $18 \%$ & $16 \%$ \\
\hline Ratio mono-/di-rhamnolipids & - & 1.67 & 1.47 & 1.67 & 1.15 & 2.37 \\
\hline
\end{tabular}

${ }^{\text {a }}$ Or Rha- $\mathrm{C}_{12}-\mathrm{C}_{10}$.

${ }^{b}$ Or Rha-Rha- $\mathrm{C}_{8}-\mathrm{C}_{10}$.

c Or Rha-Rha- $C_{12}-C_{10}$. 
et al., 2014). The size of the hydrophilic head (one or two rhamnoses) and the hydrophobic tail (one or two fatty acids), the length of the acyl chains and the presence of double bonds affect the surface-active properties of rhamnolipids. More hydrophilic congeners (di-rhamnolipids, mono-rhamnolipids with only one fatty acid, and congeners with shorter acyl chains) exhibit high cmcs (up to $200 \mathrm{mg} / \mathrm{l}$ ), whereas more hydrophobic ones (monorhamnolipids with two fatty acids and congeners with longer acyl chains) show low cmcs (between 5 and $40 \mathrm{mg} / \mathrm{l}$ ) and are more surface-active, reducing the surface tension more efficiently (Abdel-Mawgoud et al., 2009; Bharali and Konwar, 2011; Haba et al., 2003; Sarachat et al., 2010; Zhang et al., 2014). This could explain why the rhamnolipid mixtures produced in the media supplemented with OMW, which in general contain a higher proportion of more hydrophobic congeners, exhibited lower cmcs when compared with those produced in the medium CSLM.

\section{Conclusions}

In this work, rhamnolipid production by a $P$. aeruginosa strain was demonstrated using a culture medium containing as sole ingredients the agro-industrial by-products CSL, sugarcane molasses and OMW, which were used without any previous treatment. This low-cost culture medium allowed the production of $5.1 \mathrm{~g}$ of rhamnolipids per liter in reactor. Furthermore, the rhamnolipids produced exhibited a very low $\mathrm{cmc}(13 \mathrm{mg} / \mathrm{l})$. This culture medium represents a sustainable alternative for the production of rhamnolipids, and simultaneously it allows the valorization of the environmentally hazardous residue OMW.

\section{Acknowledgements}

This study was supported by the Portuguese Foundation for Science and Technology (FCT) under the scope of the strategic funding of UID/BIO/04469/2013 unit and COMPETE 2020 (POCI01-0145-FEDER-006684). The authors also thank the FCT for the financial support under the scope of the Project RECI/BBBEBI/0179/2012 (FCOMP-01-0124-FEDER-027462). E.J. Gudiña was supported by the Post-Doctoral fellowship CEB-BPD/01/2015/07 from the project UID/BIO/04469/2013, financed by FCT. A.I. Rodrigues was supported by the doctoral Grant SFRH/BD/111600/2015 provided by FCT.

\section{References}

Abdel-Mawgoud, A.M., Aboulwafa, M.M., Hassouna, N.A.H., 2009. Characterization of rhamnolipid produced by Pseudomonas aeruginosa isolate Bs20. Appl. Biochem. Biotechnol. 157, 329-345.

Abdel-Mawgoud, A.M., Lépine, F., Déziel, E., 2010. Rhamnolipids: diversity of structures, microbial origins and roles. Appl. Microbiol. Biotechnol. 86, 13231336.

Amaral, C., Lucas, M.S., Sampaio, A., Peres, J.A., Dias, A.A., Peixoto, F., Anjos, M.R., Pais, C., 2012. Biodegradation of olive mill wastewaters by a wild isolate of Candida oleophila. Int. Biodeterior. Biodegrad. 68, 45-50.

Aparna, A., Srinikethan, G., Smitha, H., 2012. Production and characterization of biosurfactant produced by a novel Pseudomonas sp. 2B. Colloids Surf. B Biointerfaces 95, 23-29.

Banat, I.M., Satpute, S.K., Cameotra, S.S., Patil, R., Nyayanit, N.V., 2014. Cost effective technologies and renewable substrates for biosurfactants production. Front. Microbiol. 5, 697.

Benincasa, M., Accorsini, F.R., 2008. Pseudomonas aeruginosa LBI production as an integrated process using the wastes from sunflower-oil refining as a substrate. Bioresour. Technol. 99, 3843-3849.

Bharali, P., Saikia, J.P., Ray, A., Konwar, B.K., 2013. Rhamnolipid (RL) from Pseudomonas aeruginosa OBP1: a novel chemotaxis and antibacterial agent. Colloids Surf. B Biointerfaces 103, 502-509.

Bharali, P., Konwar, B.K., 2011. Production and physico-chemical characterization of a biosurfactant produced by Pseudomonas aeruginosa OBP1 isolated from petroleum sludge. Appl. Biochem. Biotechnol. 164, 1444-1460.

Christova, N., Tuleva, B., Kril, A., Georgieva, M., Konstantinov, S., Terziyski, I., Nikolova, B., Stoineva, I., 2013. Chemical structure and in vitro antitumor activity of rhamnolipids from Pseudomonas aeruginosa BN10. Appl. Biochem. Biotechnol. 170, 676-689.

Dermeche, S., Nadour, M., Larroche, C., Moulti-Mati, F., Michaud, P., 2013. Olive mill wastes: biochemical characterizations and valorization strategies. Process Biochem. 48, 1532-1552.

Dubois, M., Gilles, K.A., Hamilton, J.K., Rebers, P.A., Smith, F., 1956. Colorimetric method for determination of sugars and related substances. Anal. Chem. 28, 350-356.

George, S., Jayachandran, K., 2009. Analysis of rhamnolipid biosurfactants produced through submerged fermentation using orange fruit peelings as sole carbon source. Appl. Biochem. Biotechnol. 158, 694-705.

Gonçalves, C., Lopes, M., Ferreira, J.P., Belo, I., 2009. Biological treatment of olive mil wastewater by non-conventional yeasts. Bioresour. Technol. 100, 3759-3763.

Gonçalves, C., Oliveira, F., Pereira, C., Belo, I., 2012. Fed-batch fermentation of olive mill wastewaters for lipase production. J. Chem. Technol. Biotechnol. 87, 12151218.

Gudiña, E.J., Pereira, J.F.B., Rodrigues, L.R., Coutinho, J.A.P., Teixeira, J.A., 2012 Isolation and study of microorganisms from oil samples for application in microbial enhanced oil recovery. Int. Biodeterior. Biodegrad. 68, 56-64.

Gudiña, E.J., Rodrigues, A.I., Alves, E., Domingues, M.R., Teixeira, J.A., Rodrigues, L.R. 2015. Bioconversion of agro-industrial by-products in rhamnolipids toward applications in enhanced oil recovery and bioremediation. Bioresour. Technol. 177, 87-93.

Haba, E., Pinazo, A., Jauregui, O., Espuny, M.J., Infante, M.R., Manresa, A., 2003. Physicochemical characterization and antimicrobial properties of rhamnolipids produced by Pseudomonas aeruginosa 47T2 NCBIM 40044. Biotechnol. Bioeng. $81,316-322$.

Henkel, M., Müller, M.M., Kügler, J.H., Lovaglio, R.B., Contiero, J., Syldatk, C. Hausmann, R., 2012. Rhamnolipids as biosurfactants from renewable resources: concepts for next-generation rhamnolipid production. Process Biochem. 47, $1207-1219$

Ismail, W., Shammary, S.A., El-Sayed, W.S., Obuekwe, C., El Nayal, A.M., Raheem, A.S. A., Al-Humam, A., 2015. Stimulation of rhamnolipid biosurfactants production in Pseudomonas aeruginosa AK6U by organosulfur compounds provided as sulfur sources. Biotechnol. Rep. 7, 55-63.

Kiran, G.S., Ninawe, A.S., Lipton, A.N., Pandian, V., Selvin, J., 2015. Rhamnolipid biosurfactants: evolutionary implications, applications and future prospects from untapped marine resource. Crit. Rev. Biotechnol. 24, 1-17. http://dx.doi. org/10.3109/07388551.2014.979758.

Lan, G., Fan, Q. Liu, Y., Chen, C., Li, G., Liu, Y., Yin, X., 2015. Rhamnolipid production from waste cooking oil using Pseudomonas SWP-4. Biochem. Eng. J. 101, 44-54.

Lovaglio, R.B., Silva, V.L., Ferreira, H., Hausmann, R., Contiero, J., 2015. Rhamnolipids know-how: looking for strategies for its industrial dissemination. Biotechnol. Adv. 33, 1715-1726.

Lowry, O.H., Rosebrough, N.J., Farr, A.L., Randall, R.J., 1951. Protein measurement with the Folin phenol reagent. J. Biol. Chem. 193, 265-275.

MarketsandMarkets, 2015. Surfactants Market Worth $\$ 42,120.4$ Million by 2020 . http://www.prnewswire.com/news-releases/surfactants-market-worth-421204million-by-2020-506134181.html (accessed 08.11.2015).

Meksi, N., Haddar, W., Hammami, S., Mhenni, M.F., 2012. Olive mill wastewater: a potential source of natural dyes for textile dyeing. Ind. Crop. Prod. 40, 103-109.

Mnif, I., Ghribi, D., 2015. Lipopeptides biosurfactants: mean classes and new insights for industrial, biomedical, and environmental applications. Biopolymers 104, 129-147.

Nitschke, M., Costa, S.G., Haddad, R., Gonc, L.A.G., Alves, N.C., Eberlin, M.N., et al., 2005. Oil wastes as unconventional substrates for rhamnolipid biosurfactant production by Pseudomonas aeruginosa LBI. Biotechnol. Prog. 21, 1562-1566.

Pereira, J.F.B., Gudiña, E.J., Costa, R., Vitorino, R., Teixeira, J.A., Coutinho, J.A.P. Rodrigues, L.R., 2013. Optimization and characterization of biosurfactant production by Bacillus subtilis isolates towards microbial enhanced oil recovery applications. Fuel 111, 259-268.

Perfumo, A., Rudden, M., Smyth, T.J.P., Marchant, R., Stevenson, P.S., Parry, N.J., Banat, I.M., 2013. Rhamnolipids are conserved biosurfactants molecules: implications for their biotechnological potential. Appl. Microbiol. Biotechnol. 97, 7297-7306.

Ramírez, I.M., Tsaousi, K., Rudden, M., Marchant, R., Alameda, E.J., Román, M.G. Banat, I.M., 2015. Rhamnolipid and surfactin production from olive oil mill waste as a sole carbon source. Bioresour. Technol. 198, 231-236.

Sarachat, T., Pornsunthorntawee, O., Chavadej, S., Rujiravanit, R., 2010. Purification and concentration of a rhamnolipid biosurfactant produced by Pseudomonas aeruginosa SP4 using foam fractionation. Bioresour. Technol. 101, 324-330.

Vaz, D.A., Gudiña, E.J., Alameda, E.J., Teixeira, J.A., Rodrigues, L.R., 2012. Performance of a biosurfactant produced by a Bacillus subtilis strain isolated from crude oil samples as compared to commercial chemical surfactants. Colloids Surf. B Biointerfaces 89, 167-174.

Willenbacher, J., Zwick, M., Mohr, T., Schmid, F., Syldatk, C., Hausmann, R., 2015 Evaluation of different Bacillus strains in respect of their ability to produce surfactin in a model fermentation process with integrated foam fractionation. Appl. Microbiol. Biotechnol. 98, 9623-9632.

Zhang, L., Pemberton, J.E., Maier, R.M., 2014. Effect of fatty acid substrate chain length on Pseudomonas aeruginosa ATCC 9027 monorhamnolipid yield and congener distribution. Process Biochem. 49, 989-995.

Zhang, L., Veres-Schalnat, T.A., Somogyi, A., Pemberton, J.E., Maier, R.M., 2012. Fatty acid cosubstrates provide $\beta$-oxidation precursors for rhamnolipid biosynthesis in Pseudomonas aeruginosa, as evidenced by isotope tracing and gene expression assays. Appl. Environ. Microbiol. 78, 8611-8622. 